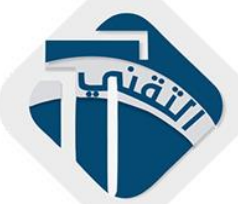

مجلة التقني

http://journal.mtu.edu.iq الموقع الألكتروني

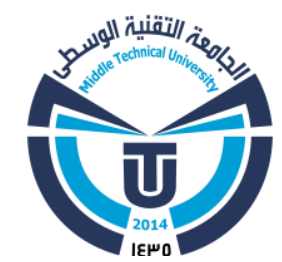

مقالة بحثية ـ التقنيات الادارية

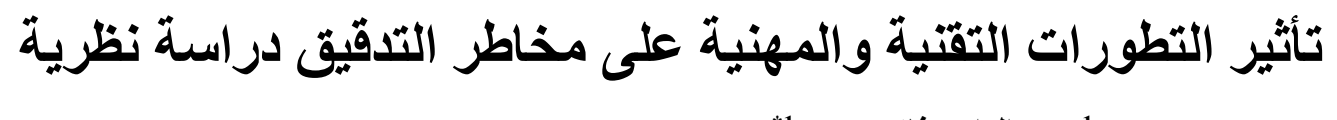

د. وحيد محمود رمو1 ، عبدالواحد غازي محمد 1*

1

abdgn1983@gmail.com البريد الأكتروني:

\begin{tabular}{|c|c|}
\hline الخلاصة & معلومات المقالة \\
\hline مو اكبة التطور رات (التقنية والمهنية) في مجال تقييم مخاطر التدقيق. & 31 اذارار 2021 \\
\hline
\end{tabular}

\title{
Impact of technical and professional developments on audit risks
}

\section{Review study}

\author{
Waheed Ramo ${ }^{1}$, Abdulwahid Ghazi Mohammed ${ }^{*}$ \\ ${ }^{1}$ Mosul University, Mosul, Iraq \\ *Corresponding author E-mail: abdgn1983@gmail.com
}

\begin{tabular}{ll}
\hline Article information & Abstract \\
\cline { 3 - 3 } Article history: & $\begin{array}{l}\text { The research provides an attempt to identify the effects of technical and professional developments on audit risks, important } \\
\text { of these developments are cloud computing, blockchain, electronic commerce, fair value and corporate governance. } \\
\text { Considering that these developments represent the modern environment for auditing in general and auditing risks in } \\
\text { peceived }\end{array}$ \\
$\begin{array}{l}\text { perticular. Therefore, the nature of these developments and their relationship to auditing and their impact on audit risks have } \\
\text { been clarified, leading to the determination of the implications of these developments on audit risks and the requirements to } \\
\text { keep up with developments (technical and professional) in the field of audit risk assessment. }\end{array}$ \\
kublished
\end{tabular}

Published

30 March 2021

Keywords: Cloud computing, blockchain, e-commerce, fair value, audit risk assessment. 


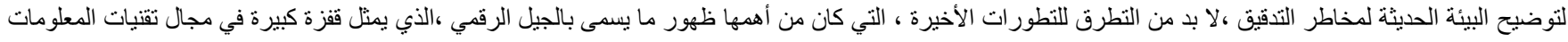

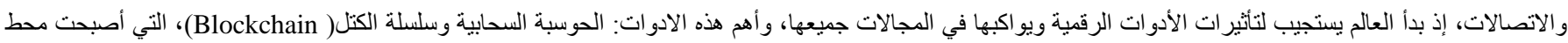

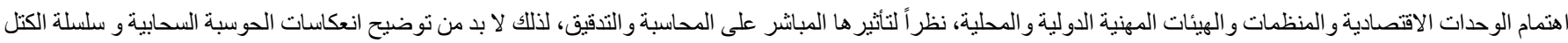

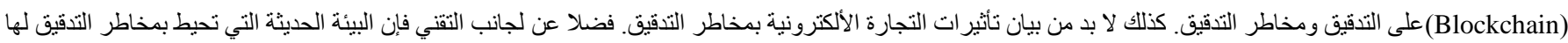

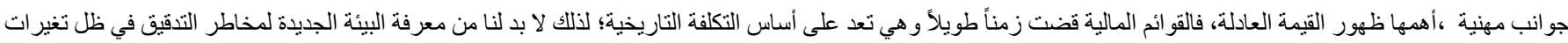
القياس المحاسبي و التي ستعتمد على القيمة العادلة كأساس للقياس والافصاح المحاسبي. والتطور المهني الآخر الذي أثر على مخاطر التدقيق الذي لا بد من الإشارة إليه، هو التعليمات

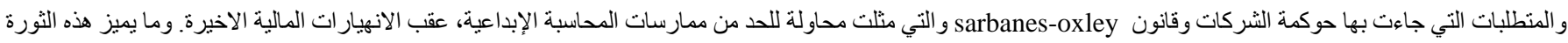
الرقمية في وقتنا الحاضر ، وجود ذلك الكم الهائل من البيانات، فتعددت وتتوعت الطر ائق و المصادر والأساليب في الحصول على البيانات و إنتاجها، وأصبحت عملية تخزين البيانات و المحافظة عليها أمر اً في منتهى السهولة، ويعزى ذلك لسهولة نشر هاوتوزيعها على مستوى الفرد والمنظمة والحكومة على الأصعدة جميعها، المحلية منها و الإقليمية و الدولية.

1.1 مشكلة البحث:

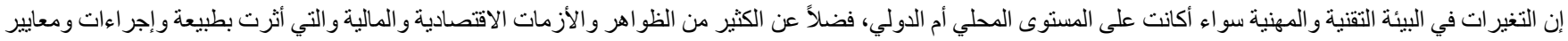

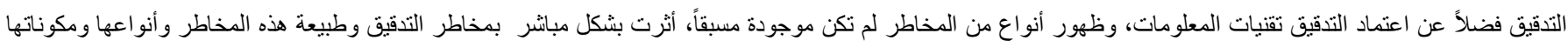
وكيفية تحديدها وتقييمها؛ ونتيجة لذلك لا بد من مو اكبة هذه التغير ات و التعرف على مخاطر التدقيق في ظل استعمال أدو ات التحول الرقمي كالحوسبة السحابية وسلسلة الكتل ماتل

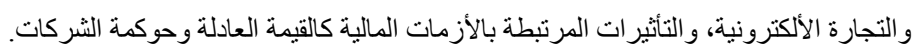
تأسيساً على ما تقدم يمكن صياغة مشكلة الدر اسة عبر الأسئلة الآتية :

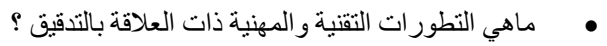

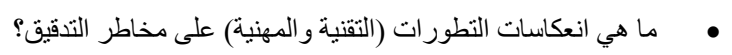
• • ماهي متطلبات مو اكبة التطور ات (التقنية والمهنية) في مجال تقييم مخاطر التدقيق؟ 1.2

تنبثق أهمية البحث من المساهمة في استجابة ومواكبة مهنة التدقيق لأهم التطورات التقنية (الحوسبة السحابية وسلسلة الكتل و التجارة الأكترونية) والمهنية (القيمة العادلة وحوكمة الثركات)، فضلا عن توفير مؤشرات للار اسات المستقبلية حول طبيعة الحوسبة السحابية وسلسلة الكتل وعلاقتهما بالتدقيق ومخاطر التدقيق، إضافة إلى المساعدة في توفير أساس للمدققين

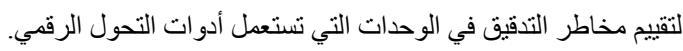

: 1.3

يسعى البحث لتحقيق الأهداف الآتية:

• معرفة علاقة التدقيق بالحوسبة السحابية وسلسلة الكتل و التجارة الألكترونية.

• توضيح تأثير التطور ات التقات التقنية ( الحوسبة السحابية وسلسلة الكتل و التجارة الألكترونية ) والمهنية ( القيمة العادلة وحوكمة الثركات ) على مخاطر التدقيق.

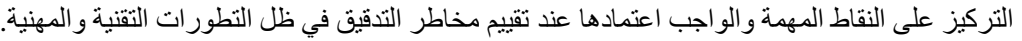

: 1.4 فرضية ومنهج البحث

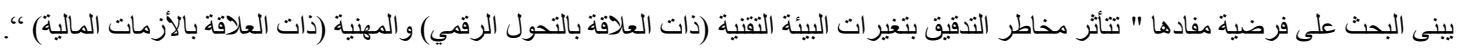

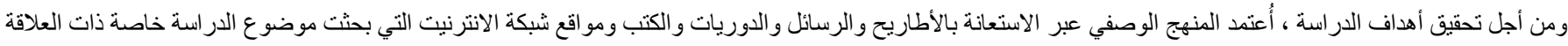

بموضو عات مخاطر التدقيق و التطور ات التقنية و المهنية . ولخروج البحث بأسلوب علمي متناسق تم تقسيمه وفقا لثلاثة مباحث أساسية على النحو الآتي :

$$
\begin{aligned}
& \text { • أهم التطور ات التقنية و المهنية وعلاقتها بالتدقيق. } \\
& \text { • • • • • • • • • } \\
& \text { • أتثير التطور ات المهنية على مخاطر التدقيق. }
\end{aligned}
$$

2. أهم التطورات التقنية والمهنية وعلاقتها بالتدقيق

2.1

تعود بدايات الحوسبة السحابية لعام 1960، إذ تنباً McCarthy بأن الحوسبة سنكون في السنوات القادمة منفعة عامة وبإمكان أي شخص الاستفادة منها وفي أي وقت. وفي منتصف

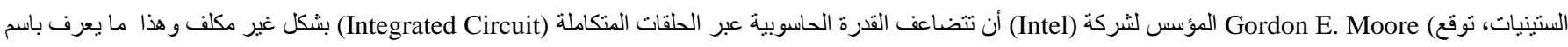


قانون (Moore ) وبحلول منتصف العقد الأول من القرن العشرين، لاحظت العديد من الوحدات الاقتصادية أن أكبر استثمار اتها في مجال تقنيات المعلومات كانت غالباً ما تظل خاملة

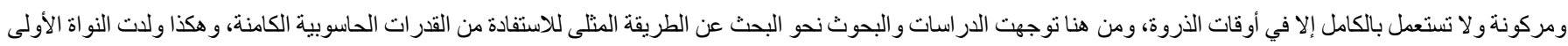
للحوسبة السحابية.

بحلول أو اخر التسعينيات، كان قانون Moore قد وجه الحوسبة إلى مستويات أعلى مما كان منوقعاً، خاصة مع تطور شبكة الويب العالمية(www) ، وفي عام 2007 تعاؤنت كل من

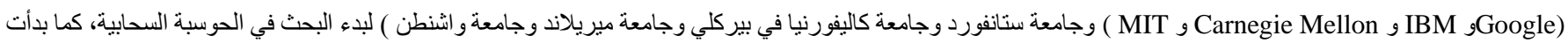

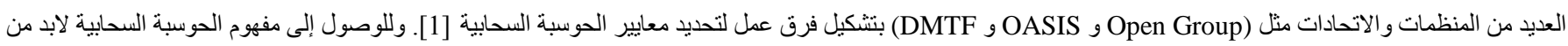
مناقثة المفاهيم المرتبطة بها والتي يمكن عبرها الوصول إلى تعريف محدد لأي نموذج للحوسبة السحابية، وأهم المفاهيم ذات العلاقة بالحوسبة السحابية: المرونة، الحيازة المتعددة،

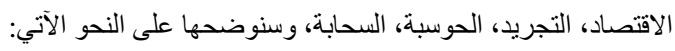

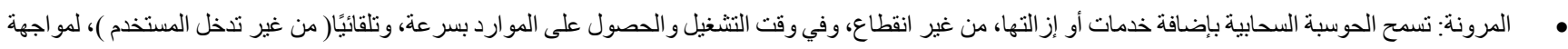

أعباء العمل، وتمثل المرونة قدرة النظام على إضافة المزيد من الموارد لتلبية متطلبات عبء العمل الكبيرة و المتز ايدة [2].

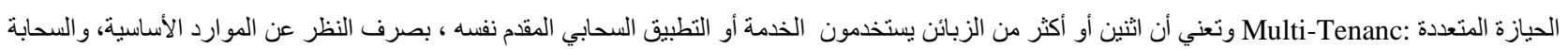

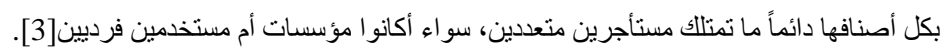

الاقتصادية: تعمل الحوسبة السحابية على توفير الموارد عبر السماح للمستهلكين باستعمال ما يحتاجون إليه فقط (بنية تحنية أوو برمجيات أو منصات)وفي الوقت المناسب، وصولاً لتوفير التكاليف.

التجريد: (Abstraction ) يقدم معظم مزودي الخدمة السحابية طبقة أو أكثر من طبقات الخدمة للمستهكين. الجانب التشغيلي لهذه الطبقات (الداعمة للخدمة) معزول عن

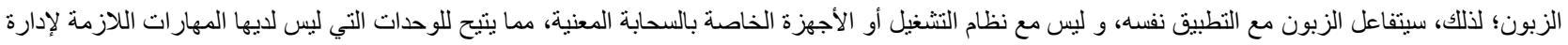

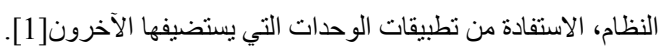

الحوسبة: هي استعمال وتوظيف وتثغيل وتظوير تقنيات الحاسوب أو أجهزته وبرمجياته من أجل القيام بمهام معينة.

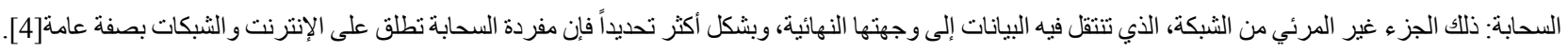

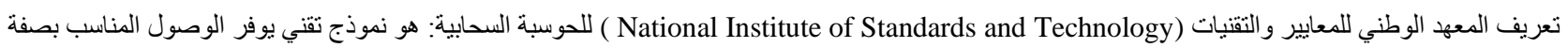

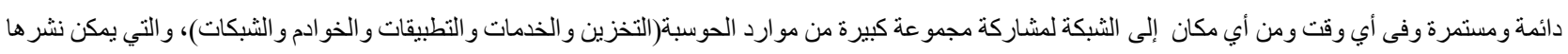

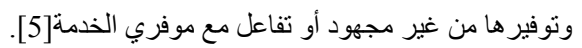

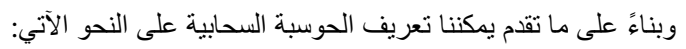

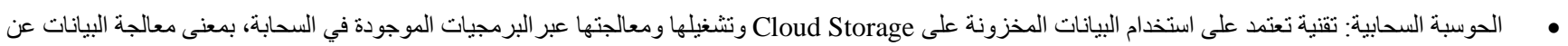

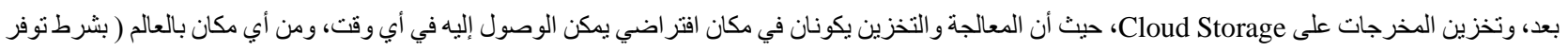

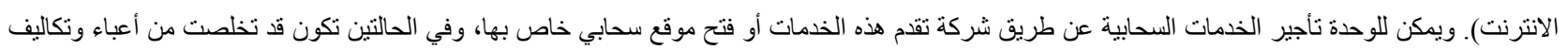
الحصول على الأجهزة و المعدات التقنية.

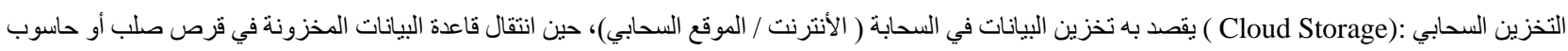
من الوحدة الإقتصادية إلى السحابة. 2.2 تمثل عملية الاستعانة بمصادر خارجية صورة للتعاقد مع جهة خارجية للقيام بأعمال قد قامت بها الوحدة مسبقاً أو للقيام بوظيفة جديدة. وتتحمل الجهة المتعاقد معها مسؤولية توفير

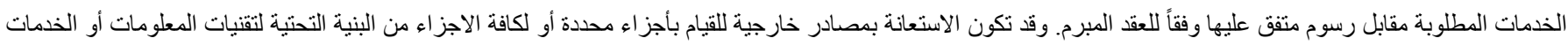

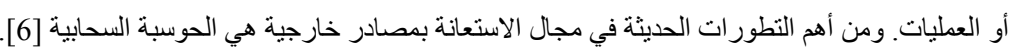
إذ انعكس استخدام الحوسبة السحابية في المحاسبة بشكل كبير على مهنة التدقيق، وذلك بسبب تز ايد المخاطر والتحديات الناتجة عن استخدام الحوسبة السحابية، والتي لا يمكن معالجتها

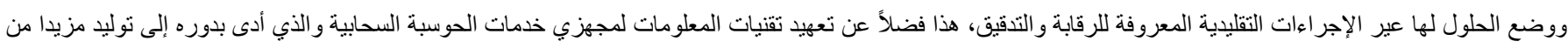

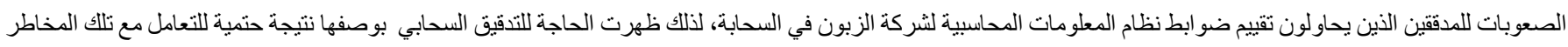

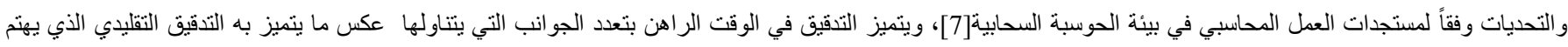
بالجانب المستندي بشكل كبير ووفقاً لذلك فإن جو انب تدقيق الحوسبة السحابية تتمثل بالآتي [8]:

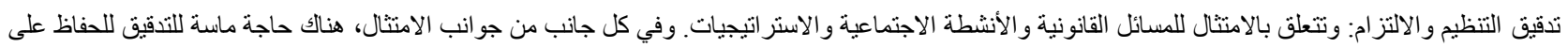

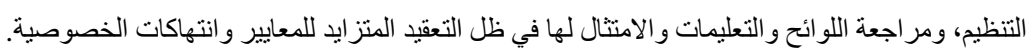

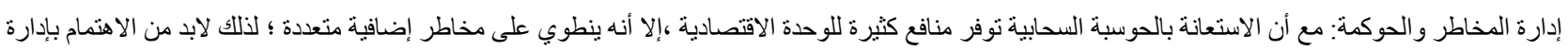
المخاطر وتعزيز الحوكمة في ظل اعتماد تقنيات المعلومات. 
Waheed R. et.al., Journal of Techniques, Vol. 3, No. 1, March 31, 2021, Pages 1- 12

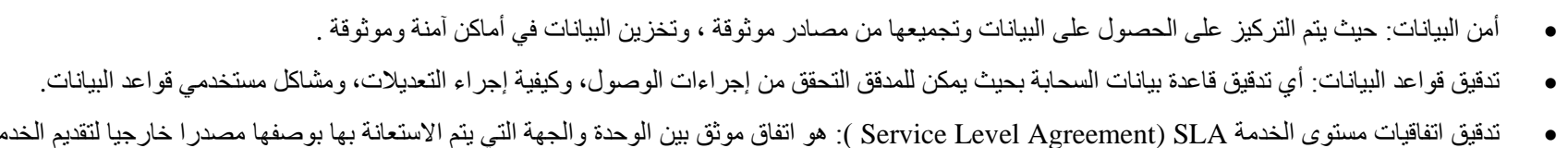

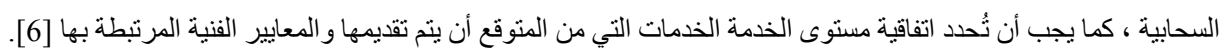

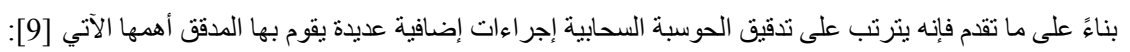

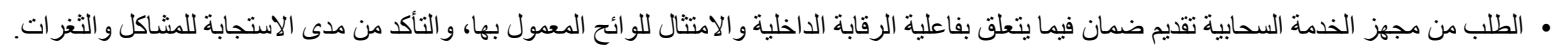
• • ت تدقيق العقود و التأكد من أنها توثث المنطلبات و المسؤوليات.

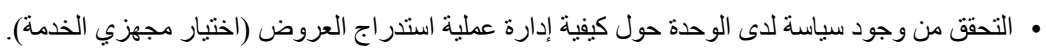
• تحديد كيفية فصل بيانات الوحدة عن بيانات الزبائن الآخرين.

• تقييم كيفية تشفير بيانات الوحدة المخزونة في السحابة.

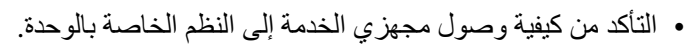

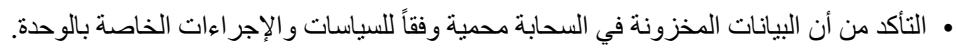
• استعر اض وتقييم الأمن المادي لمجهز الخدمة السحابية. • • استعر اض وتقييم العمليات الخاصة بتحديد كيفية مر اقبة الامتثال لاتفاقيات مستوى الخدمة. • التأكد من أن عمليات الاستعادة بعد الكوارث كافية ومناسبة لدى مجهز الخدمة السحابية . ل • تحديد إذا ما كانت عمليات الحوكمة المناسبة قائمة على مدى اشر الك موظفي الوحدة في الخدمات السحابية الجديدة. • • تقييم الخطط الخاصة بالوحدة في حال انهاء العلاقة مع مجهز الخدمة. • مر اجعة الوثائق التقييم إذاما كانت الجهة قامت بتحديد المخاطر المتعلقة بالاستعانة بمجهز خدمة أجنبي(مجهز من خارج الدولة). • تحديد كيفية الامتثال لقو انين الخصوصية المعمول بها. • مر اجعة وتقييم العمليات للتأكد من التز ام مجهز الخدمة بتر اخيص البر امج المستخدمة.

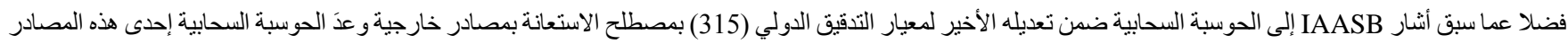
وكما يأتي: إذا كان هناك طرف ثالث (استضافة) أو استعانة بمصادر خارجية لتقنيات المعلومات، يجب على المدقق الأخذ بالحسبان إذا ما كان مجهز الخدمة كفه ومتخصص، ومر اعاة

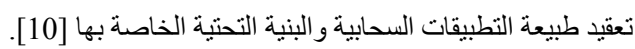
ووفقا لما تقام نلاحظ الآتي : أ. إن الحوسبة السحابية تعد خطوة تطورية وليست خطوة ثورية، بمعنى آخر ، لم تغير الحوسبة السحابية من التقنيات الموجودة بشكل كبير، ولكنها تعد نتيجة لاستخدام العديد من التقنيات الحالية و إفساح المجال للاستفادة منها بشكل أكبر و أكثر كفاءة. ب. الهدف الأساس للتدقيق في بيئة الحوسبة السحابية هو التحقق و التأكد من مدى وجود سياسة مناسبة حول الاستعانة بالحوسبة السحابية لدى الوحدة الخاضعة للتدقيق. ت. فضلاً عن مخاطر التدقيق المعروفة ينوقع أن تكون هنالك مخاطر إضافية تخص الحوسبة السحابية، وبالمقابل ستساعد برمجيات الحوسبة السحابية المدقق على زيادة دقة عمله وبالنتيجة تسهيل عملية تحديد مخاطر التدقيق.

2.3 سلسلة الكتل Blockchain (المفهوم، التعريف، الخصائص، الاستخدامات، التأثبر على التدقيق):

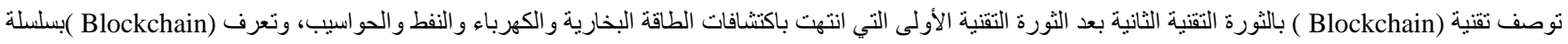
الكتل أو سلاسل التقة وكان الاستخدام الأول لها عام 2008، وتمثل هذه التقنية البنية التحتية لتداول العملات الافتراضية، و من أسباب ظهور هذه التقنية هو البحث عن ابتكار لحل مشكلة انعدام الثقة عند إجر اء المعاملات بين طرفين مجهولين من غير الحاجة إلى طرف ثالث وسيط بلان بينهما. ووفقا لإحصاءات منظمة التجارة العالمية فان المستقبل القريب سيشهد استثمارات

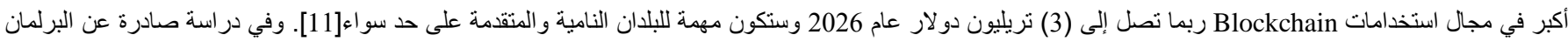

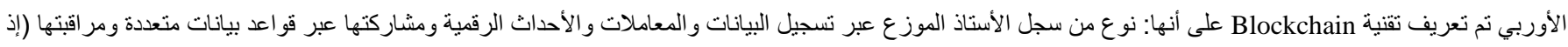

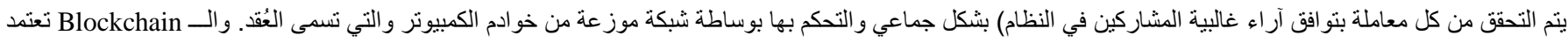

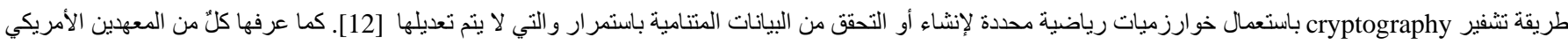
و الكندي للمحاسبين القانويين على أنها: سجل الأستاذ الرقمي الذي تم إنشاؤه لتسجيل المعاملات التي تتم بين مختلف الأطر اف في الثبكة. و الذي يستند على أساه النس الند للند، و المشاركين

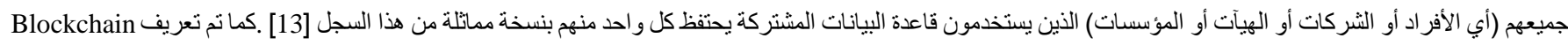
على أنه أكبر سجل رقمي موزع ومفتوح يسمح بنقل أصل الملكية من طرف إلى آخر في الوقت نفسه (Real Time) من غير الحاجة لوسيط، مع تحقيق درجة عالية من الأمان لعطلية

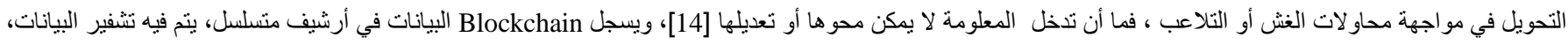
بحيث لا يمكن التلاعب في المدخلات الأصلية، كما يمكن مشاركتها والوصول إليها من قبل أب شخص لديه شفرة الدخول، ويتم تجميع المعاملات وتحديثها كل عشر دقائق ثقريبًا، فيصبح 
Waheed R. et.al., Journal of Techniques, Vol. 3, No. 1, March 31, 2021, Pages 1- 12

شيبًا آخر في كل مرة يتم تجديدها، وعندما يريد شخص ما إضافة معاملة جديدة، سيتحقق المشاركين في الثبكة جميعهم من صحة تلك المعاملة، من غير الحاجة لسلطة مركزية، ثم بترك

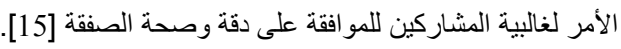

ومن ثم يمكننا تعريف (Blockchain) بأنه سجل رقمي يمكن عبره إجر اء المعاملات كافة ونقل الملكية وتبادل العملات الرقمية بدرجة عالية من الثفافية و الأمان للأفر ادو الثركات

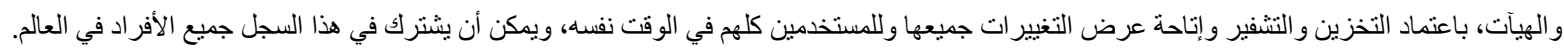

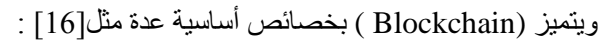

• النشر: حيث يمكن للمشاركين جميعهم الوصول إلى نسخة كاملة من دفتر الأستاذ وبنسخ منطابقة ومتكافئة، ولا يوجد طرف لديه سيطرة على دفتر الأستاذ، حيث ينم نشر المعاملات الجديدة بسر عة ويتم نشر ها على نسخ المشاركين جميعا.

الاستمر ارية: حيث أن كل مستخدم لايه نسخة خاصة به من دفتر الأستاذ، وتتحدد الثقة بينهم بالإجماع، ولا يمكن تعديل المعاملات السابقة من غير موافقة الأغلبية، مما بعني

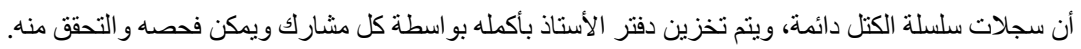
البرمجة: تسمح بعض سلاسل الكتل بتخزين رمز البرنامج عليها، وكذلك الترحيل إلى دفتر الأستاذ يتم تنفيذه تلقائيًا من القيود اليومية التلقائية التي تسجل عند التشغيل، وهذه ونده العملية تسمى ب "العقود الذكية."

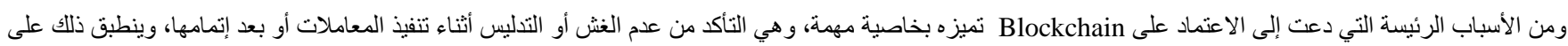

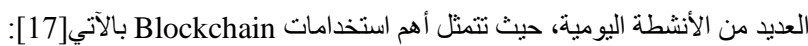

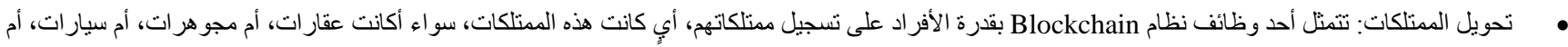
بر اءات اختر اع وحقوق ملكية فكرية وغير ها بحيث يستطيع الأفر اد بعد ذلك بيعها عبر نظام Blockchain ، أو إجر اء معاملات عليها فيما بعد.

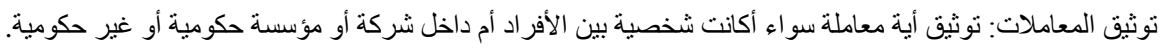

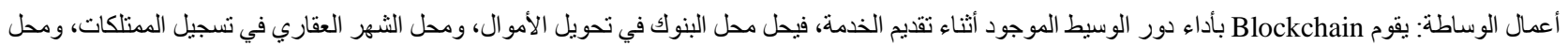
إدار ات المرور في تسجيل السيار ات، ومحل السماسرة في عمليات البيع والثراء ومحل الثركات الوسيطة، وذللك لصالح وسيط جديد هو ملايين الأفراد حول العالم الذين

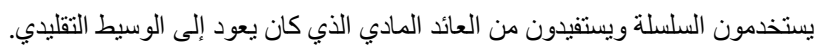

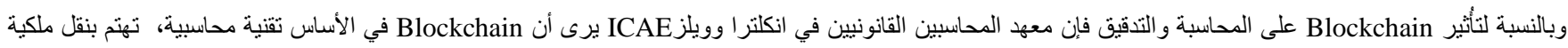
الأصول و الحفاظ على المعلومات المالية الدقيقة المدرجة بدفتر الأستاذ، وبما إن مهنة المحاسبة تهتم على نطاق واسع بقياس المعلومات المالية وإيصالهاوتحليلها وقياس الحقوق والالتزامات على الممتلكات، فإن استخدام Blockchain يوفر الثفافية بشأن ملكية الأصول، كما أن لايه قدرة تعزيز مهنة المحاسبة عبر تقليل تكاليف صيانة وتسويات دفتر الأستاذ ونوفير الثقة و الموضو عية العالية بشأن ملكية الأصول[16]، ويمكننا عرض التأثير ات المتوقعة للـ Blockchain على التدقيق عبر الجو انب الآتية[18]: ظهور خدمات جديدة في التدقيق: ستؤدي تقنية Blockchain إلى ظهور جيل جديد من خدمات التدقيق عبر المز اياو التحديات المر افقة لاستخدامها، مما يجعل المدققين مطالبين

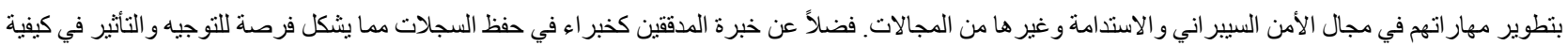
تضمين هذه التقنية واستخدامها في المستقبل، وتطوير الحلول و الخدمات المرتبطة بها. التشفير وعدم قابلية المعاملات للتعديل أو التغيير: هذه الخاصية التي يوفرها Blockchain تجعل عملية التحقق من المعلومات المرتبطة بالبيانات المالية تلقائية باستعمال البرمجيات المخصصة للتدقيق . تقليل وقت وتكلفة خدمات التدقيق: إذ أن أتمنة وظائف التدقيق ستقلل بشكل كبير من وقت ونكلفة خدمات التدقيق. المزيد من الثفافية: وذلك لأن إتاحة رؤية المستخدمين المعتمدين للمعاملات جميعها، يقلل من إجر اءات عمل المدققين خاصة فيما يتعلق بأخذ العينات والتحقق من المعاملات، وبالنتيجة يتيح للمدققين مزيدا من الوقت للتركيز على الضو ابطو التحقيق في العمليات الثاذة. مسار ات التدقيق التي يمكن تتبعها: سنوفر تقتية Blockchain مسار ات تدقيق يمكن تتبعها، عبر أتمتة عمليات التدقيق، و المصادقة السريعة للمعاملات، و التتبع السريع لملكية الأصول. التأثير بأدوار المدققين: Blockchain طريقة عمل المدققين، من حيث الأشخاص الذين سيعملون معهم وأماكن القيام بوظائفهم، وكيفية التواصل مع الزبائن و الزملاء. التأثير بمسؤوليات المدقق: تضيف تقنية Blockchain مسؤوليات أخرى للمدقق و أهم هذه المسؤوليات ما يأتي: ضرورة التحقق من الأصول الرقمية للزبون محل التدقيق،

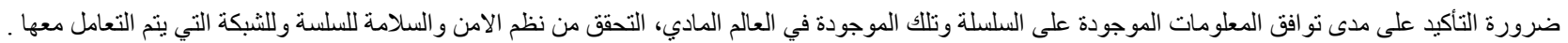
مسؤولية منع أية معاملات غير صحيحة من حفظها في الكتلة الرقية الخاصة بها ، مسؤوليته عن اشتمال تقرير التدقيق كل المعلومات المهمة عن نظام المعلومات بالوحدة

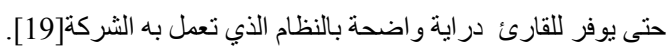
التهايدات و المخاطر : مع كل الإمكانيات و المز ايا التي توفر ها تقتية Blockchain إلا أن ذلك سير افقه بعض التهديدات خاصة بالنسبة للمحاسبة والتدقيق[20]. 
2.4

إن تز ايد معدلات التجارة الألكترونية فرض على على التئن المدققين تقديم خدمات جديدة بغرض إضفاء الثقة على معاملات الوحدة الاقتصادية التي نتم في إطار التجارة الألكترونية، فضلاً عن

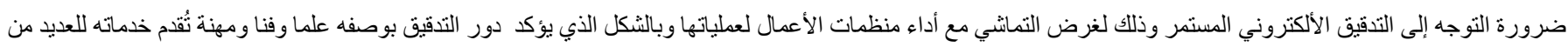

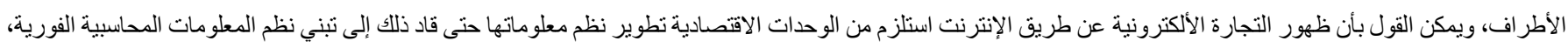

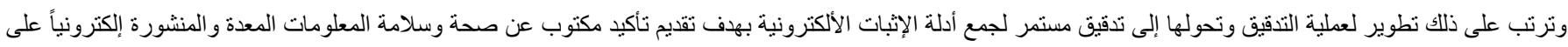

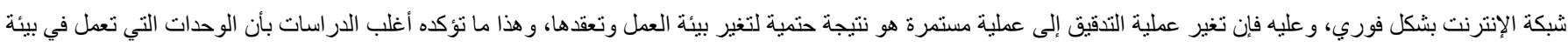

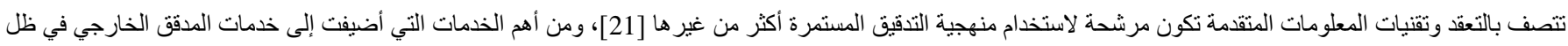
التجارة الأكترونية [22]:

خدمات توكيد الثقة في المو اقع الأكترونية : هي عبارة عن تصديق من أحد مكاتب المحاسبة والتدقيق على تأكيد الإدارة، يؤكد بدوره للزبائن أن تطبيقات

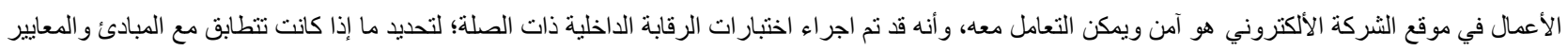

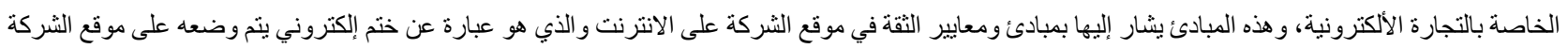

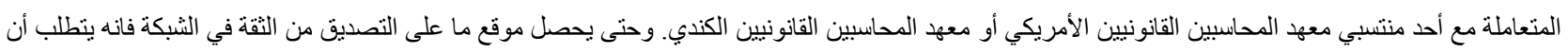
يكون نشاط الموقع معروفاً بالإضافة إلى ضرورة وجود إجر اءات رقابية للحفاظ على جودة الموقع وللتأكد من إن معلومات الزبون آمنة ومحمية من أي استخدام غير قانوني.

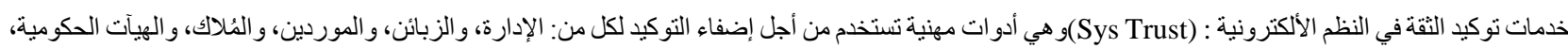

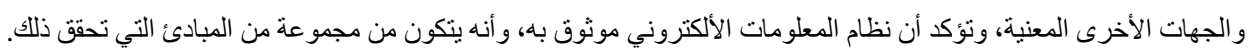

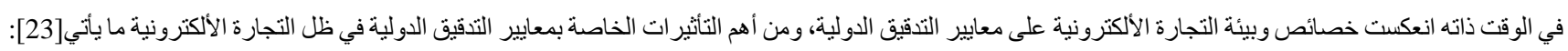

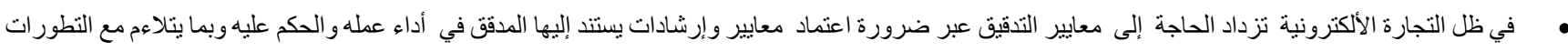

التقنية التي استجدت في هذا المجال.

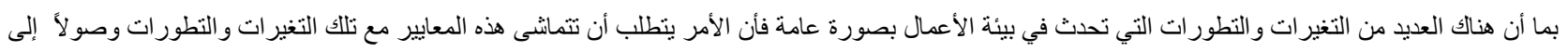

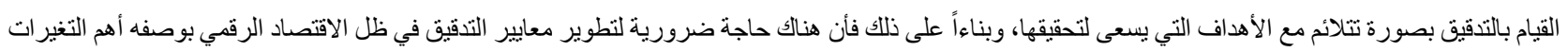

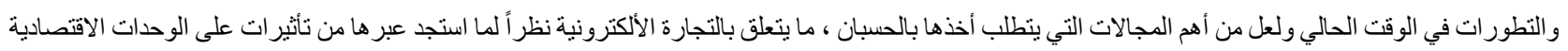

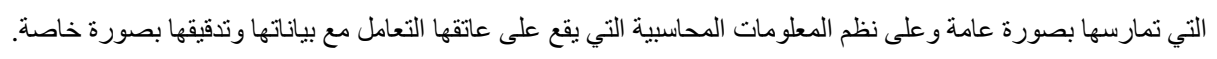

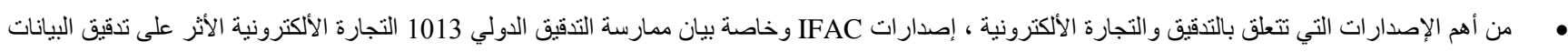

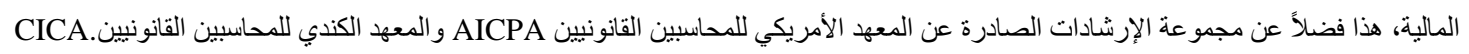

2.5 تدقيق القيهة العادلة:

تتعلق عملية تدقيق القيمة العادلة بتدقيق قياسات و إفصاحات القيمة العادلة وفقاً لمعايير التدقيق الدولية، وذلك من أجل تحقيق أهداف عدة أهمها [24]:

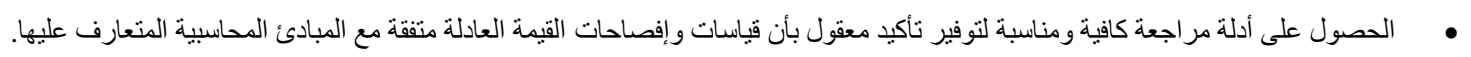

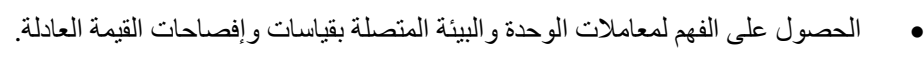

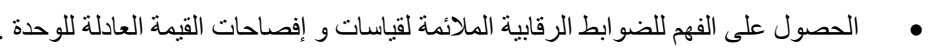

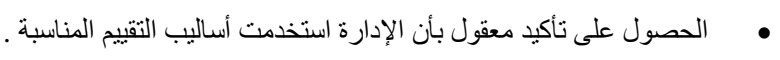

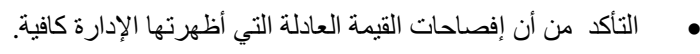
2.6 نأثبر حوكمة الشركات على التنقيق في الوقت الذي يعد فيه كل من التدقيق و الرقابة الداخلية أدوات مهمة من أدو ات تطبيق حوكمة الثركات، فإننا يجب أن نعلم أن الحوكمة ساهمت أيضاً في تفعيل كل من التدقيق الخارجي

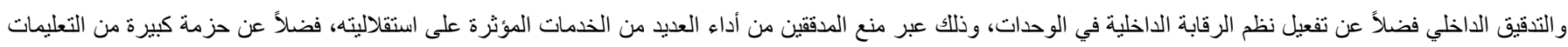
الرقابية المؤثرة و الفاعلة.

3. تأثير أهم التطورات التقنية على مخاطر التدقيق

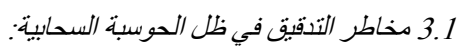

3.1.1 أسباب مخاطر التدقيق في ظل الحوسبة السحابية:

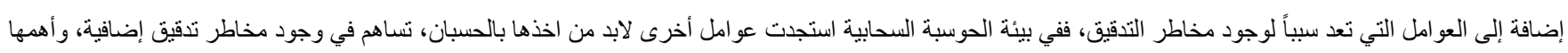


Waheed R. et.al., Journal of Techniques, Vol. 3, No. 1, March 31, 2021, Pages $1-12$

خسارة المعرفة المتعلقة بالأعمال، لأن تطوير التطبيق سيتم خارج الوحدة، لذلك فإن الوحدة تتعرض أيضاً لخطر فقدان ملكية منهجية الأعمال، والتي قد يطالب بها مجهز

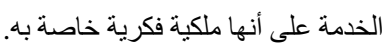

فثل مجهز الخدمة من حيث عدم تو افق الخدمات التي يتم تحصيلها مع احتياجات المستخدمين، أو إنها تكون دون المستوى، أو ذات تكلفة مرتفعة. كذلك بالنسبة لتوظيف مجهزي الخدمة من خارج الدولة، فالمخاطر تزداد مع وجود قو انين أجنبية حول تخزين ونقل المعلومات، مما ينعكس على خصوصية البيانات وحمايتها في حال عدم وجود قو انين أجنبية تناسب طبيعة الوحدة. 3.1 .2 مخاطر التدقيق المستجدة في بيئة الحوسبة السحابية:

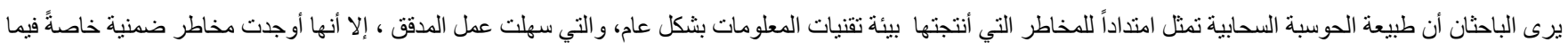
يتعلق باختفاء السجلات وصعوبة تتبع الدليل الألكتروني، ومخاطر الرقابة فيما يخص تعامل الرقابة الداخلية مع إجراءات جديدة ترتبط بكيفية التعامل مع مجهز الخدمة السحابية ومتابعة

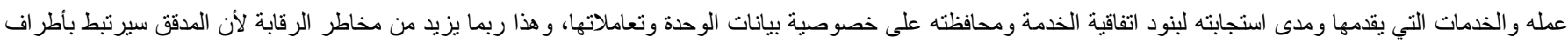

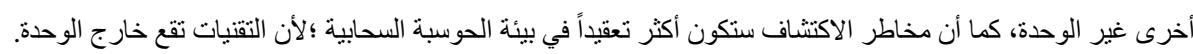

3.1 .3 الا هتمام بالتأهيل الدهني للمدقق لمواجهة مخاطر التدقيق في ظل الحوسبة السحابية:

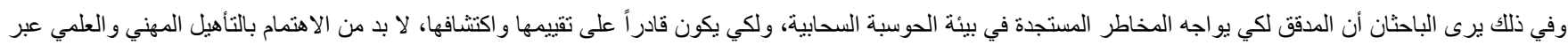

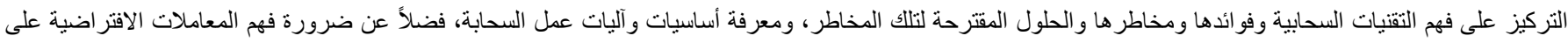

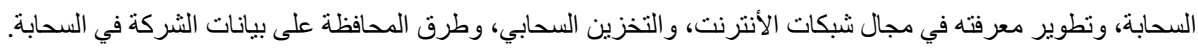
3.1 .4 إذ يلاحظ الباحثان أن الوجه الآخر للحوسبة السحابية هو دور ها في تسهيل عملية التدقيق وبالنتيجة مساعدة المدقق في تسهيل عملية تحديد مخاطر التدقيق، عبر الاستفادة من برمجيات

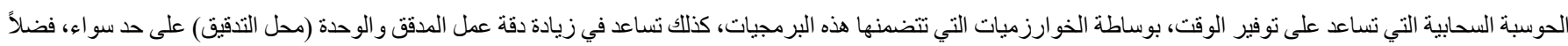
عن إنها تساعد المدقق و الوحدة على نشر نتائج التدقيق و التقارير المالية عبر اجتماعات افتراضية باستعمال السحابة مع إمكانية اتساع قاعدة المستفيدين من هذه التقارير. 3.2 يمكننا تحديد تأثيرات Blockchain بمخاطر التدقيق على النحو الآتي : إن الاستفادة و المز ايا التي يقدمها Blockchain للمدقق، تساهم في توفير تقارير تدقيق أكثر دقة كما ستساعد في انخفاض مخاطر التدقيق، عبر قيام Blockchain بالتحقق

$$
\text { التلقائي للعديد من المعاملات بوقت قياسي. }
$$
مع أن تقنية Blockchain تضمن شفافية المعاملات وعدم قابليتها للتر اجع عن أية معاملة إلا أنه لا يمكنها القضاء على حالات الاحنيال بشكل كامل، إذ يعتد التبني الناجح

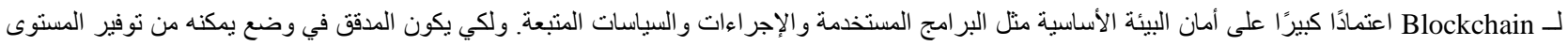

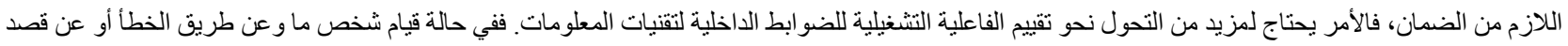

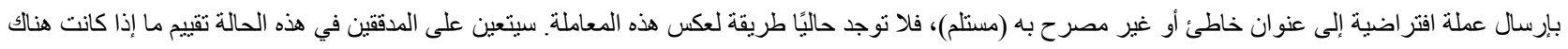

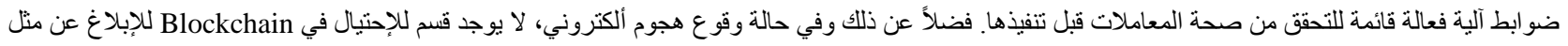

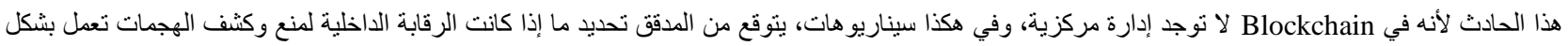
فعال. بالإضافة إلى ذلك، في حالة فقدان مفتاح خاص بسبب عطل في أحد البرامج أو الأجهزة، سيتم فقدان الوصول إلى أبة عملة افتراضية (مثل البيتكوين) مرتبطة بهذا

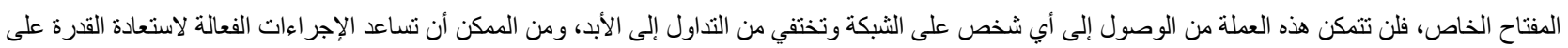
العمل بعد الكو ارث وإجر اءات النسخ الاحتياطي في منع حدوث مثل هذه الحالات [25]. ونلاحظ أن توفر خاصية عدم التراجع عن معاملة ما في Blockchain ستكون

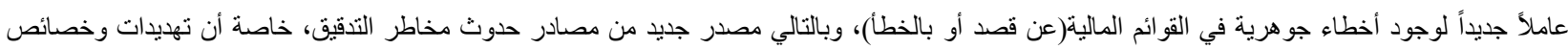
Blockchain في الوقت الحالي تمثل أمور اً غير مألوفة لاى المدققين، وحتى لدى التقنيين الذين ما زالو ا بعملون على تطوير إمكانيات Blockchain وتقليل الثغرات فيه. بما أن مستخدمي تقنيات Blockchain سيو اجهون مخاطر حدوث أخطاء أو نقاط ضعف مجهولة الهوية، فقد يحتاج المدقق إلى مجمو عة مهار ات جديدة، بما في ذلك فهم لغة البرمجة التقنية ووظائف Blockchain ، للقيام بأدوار جديدة لمو اجهة مخاطر التدقيق المحتملة نتيجة لتطبيقB Blockchain ، ويثير هذا النوع من الأدوار أيضًا أسئلة مهمة

لمهنة التدقيق، منها [13]:

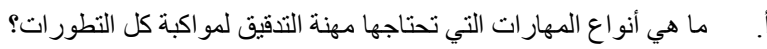

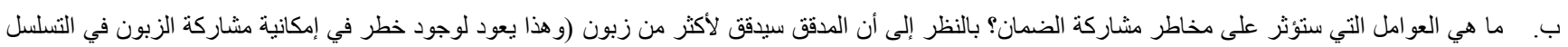

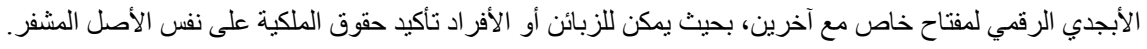

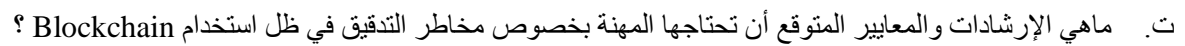


Waheed R. et.al., Journal of Techniques, Vol. 3, No. 1, March 31, 2021, Pages 1 - 12

ور غم الميز ات التي يوفر ها Blockchain لكن هنالك حاجة لطرف ثالث موثوق به ومستقل لتقديم ضمانات بشأن فاعلية الضو ابط على سلسلة المفاتيح الخاصةBlockchain ، و هذا النوع .

من الخدمة يثير أسئلة مهمة للمهنة :

1

2.

• • المخاطر الضمنية المرتبطة بالـ Blockchain وا يتعلق بتعقيد شبكة Blockchain والتي يمكن أن تختلف اختلافًا كبيرًا اعتمادًا على عدد العقد على الثبكة، ووجود أو

عدم وجود عقد احتياطية، وحكم الإدارة فيما إذا كانت هناك حاجة إلى أنظمة النسخ الاحتياطي، ففي شبكة مكونة من عدة آلاف من المستخدمين المنتشرين على مساحة واسعة،

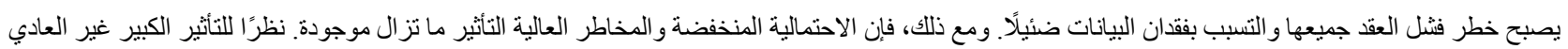

الذي يمكن أن يحدثه فشل الثبكة في عملية تجارية قائمة على Blockchain ، فلذك يتعين معالجة مجال هذه المخاطر [26]. فيما يتعلق بتدقيق العملات الافتر اضية فقد حدد المعهد الكندي للمحاسبين القانونيين أمثلة للظروف و الأحداث التي من المحتمل أن ينظر فيها المدقق كجز ع من تتفيذ الإجر اءات

لتحديد وتقييم مخاطر الأخطاء الجو هرية في معاملات وأرصدة العملات الافتر اضية سو اء أكان ذلك بسبب الاحتيال أم الخطأ [27] :

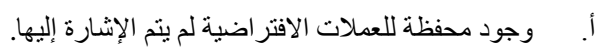

ب. فقدان المفتاح الخاص وبالنتيجة لم يعد بالإمكان الوصول إلى العملة الافتر اضية ذات الصلة.

ت. يحصل الطرف غير المصرح به على المفتاح الخاص ويسرق العملة الافتر اضية الخاصة بالوحدة.

ث. إرسال العملة الافتراضية إلى عنوان غير صحيح ولا يمكن استرداد العملة الافتر اضية.

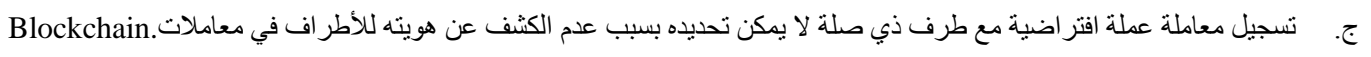

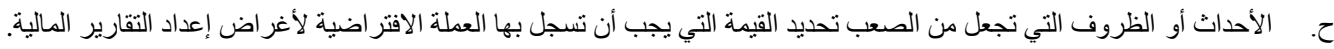

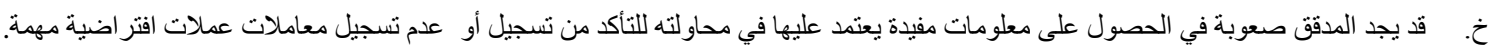

• سيكون من الصعب على المدققين تقييم ما إذا كانت الإدارة قد حددت بشكل صحيح و أفصحت عن عمليات العملات الافتراضية جميعها مع الأطر اف ذات العلاقة، وسيكون من الصعب الحصول على أدلة تدقيق مناسبة كافية عندما لا يكون لدى الوحدة ضو ابط داخلية فعالة لتحديد عمليات العملات الافتر اضية مع الأطر اف ذات العلاقة، وتمثل هذه

النقطة مجالا و اسعا لمخاطر التدقيق.

• فيما يتعلق بالوحدات التي تقيس العملات الافتر اضية بالقيمة العادلة، فمن المرجح أن يتم تقييمها على أنها مخاطر كبيرة من قبل المدققين، خاصة فيما يتعلق بتقييم المدققين

لوجود سوق نشط ومماتل للعملات الافتر اضية[28].

و استجابة للتطورات التي رافقت استخدام الـ blockchain أشار IAASB في التعديل الأخير الصادر نهاية عام 2019 لمعيار التدقيق الدولي 315 إلى التعامل مع التقنيات الحديثة

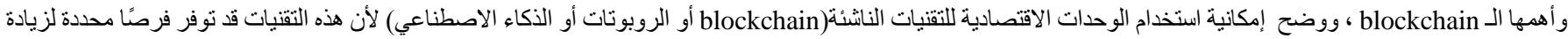

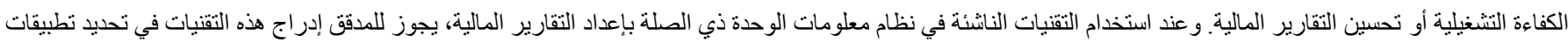
تقنيات المعلومات و الجو انب الأخرى لبيئة تقنيات المعلومات التي تخضع للمخاطر الناشئة عن استخدام تقنيات المعلومات. وينبغي مر اعاة أن التقنيات الناشئة قد تكون أكثر تطور أ أو تعقيدًا مقارنة بالتقنيات الحالية [10]، مما يؤكد أن هذه التقنيات تعد بيئة جديدة لمخاطر التدقيق، مع إمكانية أن تكون أكثر تعقيداً ومن ثم تكون هنالك مخاطر جديدة واحتمالية ارتفاع مستوى ونئي مخاطر التدقيق خاصة في السنو ات الأولى لتبني هكذا تقنيات.

3.3 تأثبير التجارة الألكترونية على مخاطر التدقيق:

• المخاطر الضمنية في ظل التجارة الألكترونية: نظر اً لطبيعة أنشطة التجارة الأكترونية، واعتمادها مستوى متقدم من تقنيات المعلومات والاتصالات، فإن المخاطر الضمنية

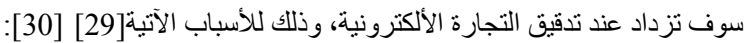

1. صعوبة تتبع أدلة التدقيق الأكترونية: في نظم التجارة الألكترونية، ينم نقل الوثائق الأصلية مثل الفو اتير و الثنيكات وطلبات المواد و إدخالات التخزين عبر الإنترنت، مما يؤدي

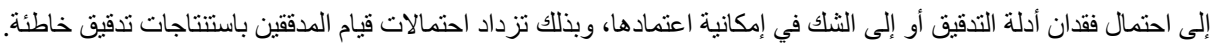

2. الاختر اقات و الهجمات التي تتعرض لها البيانات الألكترونية: بيئة التجارة الألكترونية تعد بيئة مفتوحة واحتمالية تعرض البيانات فيها لخطر التشويه والضياع واردة جداً،

فضلاً عن أن التوقيع الألكتروني سيكون مختلفاً عن البدوي من الناحية الفنية و القانونية .

3. اعتماد الوحدة طرف ثالث في إتمام الكثير من معاملاتها، وحدوث أية اختر اقات أمنية، أو أخطاء أو تلاعب في نظام الطرف الثالث، من المكن أن يؤدي إلى نتائج سيئة على

أنشطة الوحدة.

و التأثثرات السابقة من شأنها أن تزيد مستوى المخاطر الضمنية من ناحية، و خطر الأعمال من ناحية أخرى، الأمر الذي يؤدي إلى زيادة مستوى خطر التدقيق الكلي، و من أجل تقدير

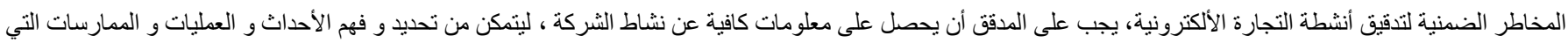
قد يكون لها أثر على التقارير المالية أو على تقديره للمخاطر الضمنية. كما لابد أن يمنلك مهار ات مناسبة في تقنيات المعلومات والأنترنت ليقوم باستفسارات عدة مهمة عن النشاط و استر اتيجية الوحدة في التجارة الأكترونية، و التقنيات اللازمة لإتمام التجارة الأكترونية، فضلا عن إمكانية اللجوء إلى خبير للمساعدة في تقييم مدى دقة إجر اءات الأمن و الحماية لتقنيات

المعلومات ووسائل الرقابة الداخلية [30]. 
Waheed R. et.al., Journal of Techniques, Vol. 3, No. 1, March 31, 2021, Pages 1- 12

ونلاحظ أنه مع تطور تقنيات المعلومات و الاتصالات، واعتماد المشروعات الوسائل التقنية الحديثة في إتمام معاملاتها، برى الكثيرون ان ذلك هو السبب في زيادة احتمال وجود

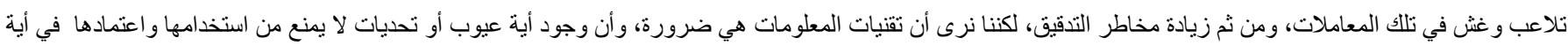

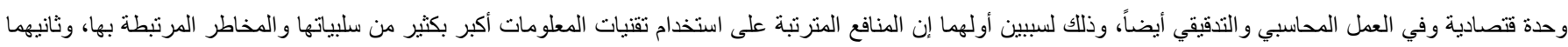

الإمكانية الحتمية في التغلب على الثغرات المصاحبة لاستخدامها تدريجياً.

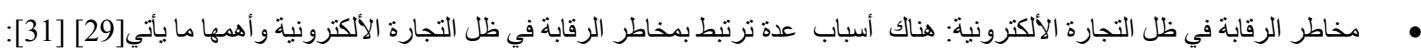

1. آليات تقسيم عمل خارج نطاق الرقابة: في ظل اعتماد تقنيات المعلومات ستكون بعض الوظائف و الصلاحيات مرتبطة بكلمات مرور، أب أن تقسيم العمل سيعتمد رموزا

ألكترونية، وهنا قد يحصل بعض الأشخاص على كلمات مرور تقع خارج نطاق سلطتهم بشكل غير قانوني، مما يجعل بعضهم يقدمون فو اتير وأو امر مزيفة.

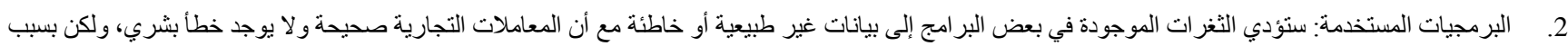

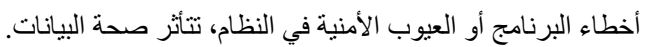

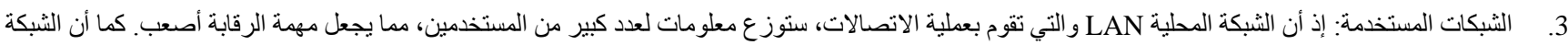

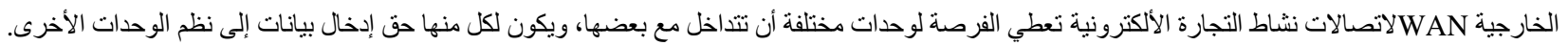

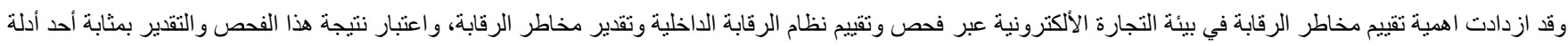

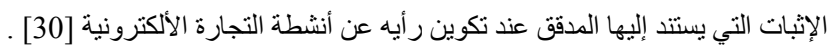

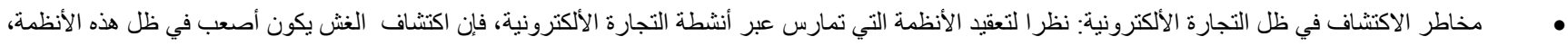

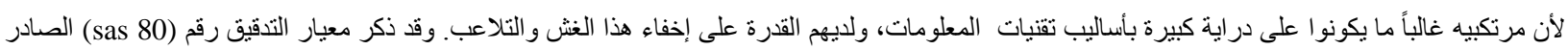

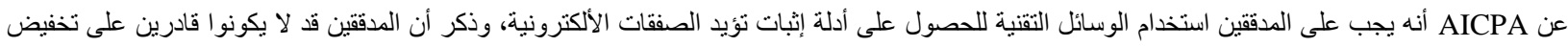
مخاطر الاكتشاف للمستوى المقبول أو المتعارف عليه عن طريق أداء الاختبار ات الأساسية فقط لهذه النظم المتقدمة، لذلك لابد للمدقق من أختبار أساليب الرقابة المتبعة على هذه النظم [30]. ونلاحظ هنا أهمية قيام المدقق بالتحقق من أمان وموثوقية نظام التجارة الأكترونية، خاصة مع تحديث إصدار البرنامج سنوياً أو شهرياً، وعدم الاكتفاء بالتحقق من صحة وشرعية البيانات التي ظهرت في أنشطة التجارة الإكترونية.

4. تأثير التطورات المهنية على مخاطر التدقيق

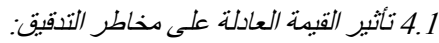

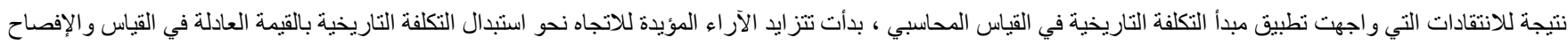
المحاسبي، و استجابة لهذه الآر اء تبنت المعايير الدولية القيمة العادلة كأساس للقياس المحاسبي للكثير من الأصول و الالتزامات، مما ساهم في خلق تحديات قد تواجه المدققين بشأن تدقيق

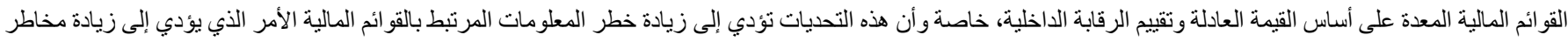

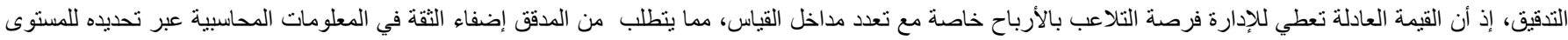

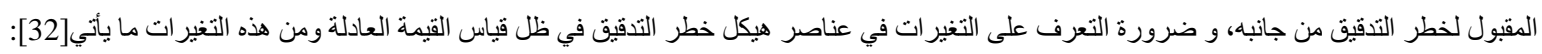

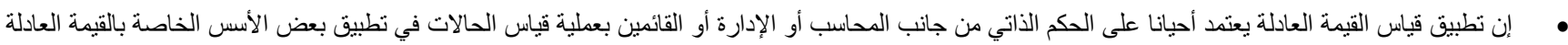

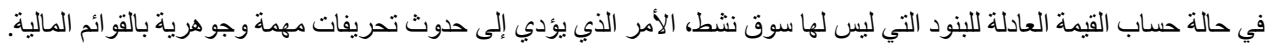
إن تطبيق قياس القيمة العادلة وما يتبعه من معالجات محاسبية في القياس و العرض و الإفصاح قد يسبب تحريفات مهمة وجو هرية بالقو ائم المالية، وذللك بسبب عدم استخدام أساس القياس المناسب خاصة في حالة عدم وجود سوق نشط، أو عدم إتباع أسلوب العرض و الإفصاح المناسب، أو حتى بسبب أخطاء في العمليات الحسابية. إن تطبيق أساس القيمة العادلة يتطلب التحقق من مدى كفاية ومناسبة الإفصاح الإضافي عن الطرق و الافتر اضات الجو هرية المستخدمة في تحديد القيمة العادلة، و التي قد تمثل مجالاً خصباً لوجود تحريفات مهمة وجو هرية بالقو ائم المالية.

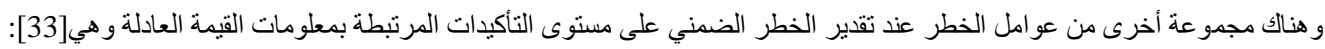
عو امل خطر مرنبطة بالبيانات والمعلومات المستخدمة في القياس فكلما ابتعدت هذه البيانات و المعلومات عن السوق وارتبطت بالظروف الداخلية للوحدة والآراء الثخصية للقائمين على القياس ،ز ادت المخاطر الضمنية. عو امل خطر مرتبطة بأساس قياس القيمة العادلة الذي يعتمد في بعض الأحيان على الأسعار المعلنة بالأسو اق النشطة وفي الكثير من الأحيان على نماذج وطرق قياس معقدة و افتراضات وبيانات قد تعتمد التقدير الثخصي وتختلف في درجة دقتها وموضوعيتها وملاءمتها ، بالنتيجة تنخفض المخاطر الضمنية كلما كان القياس معتمدا الأسعار المتداولة في الأسواق النشطة ، وتزداد في حالة اعتماد نماذج وطرق التسعير في حالة الأسو اق غير النشطة. عو امل خطر مرتبطة بنماذج وطرق القياس في حالة الأسو اق غير النشطة فكلما كانت هذه النماذج والطرق تعتمد الأساليب الرياضية والإحصائية كلما انخفض الخطر الضني في وفي و العكس صحيح. عو امل خطر مرتبطة بمعايير المحاسبة عن القيمة العادلة فكلماز ادت تعقيدات هذه المعايير سو اء أكانت مرتبطة بالقياس أم الإفصاح ،ز ادت المخاطر الضمنية. 
Waheed R. et.al., Journal of Techniques, Vol. 3, No. 1, March 31, 2021, Pages 1- 12

عو امل خطر مرتبطة بالقائمين بقياس القيمة العادلة فكلما انخفضت مهار ات وكفاءة القائمين بقياس القيمة العادلة ، زادت المخاطر الضمنية . عو امل خطر مرتبطة باستعمال عمل الخبير في قياس القيمة العادلة فكلما كان الخبير ذو تأهيل وخبرة ومهار ات مناسبة و لا توجد علاقات مصالح بينه و بين الوحدة ، انخفضت

المخاطر الضمنية و العكس بالعكس .

وتأسيساً على ما سبق يمكن أن نلاحظ الآتي :

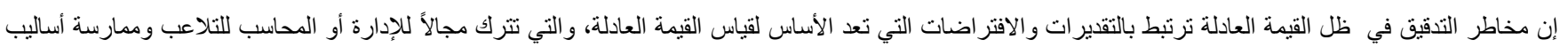
المحاسبة الإبداعية بصورة تؤثر باحتمالية زيادة مخاطر التدقيق ووجود مخاطر جديدة، مع أن القيمة العادلة تساهم في وجود معلومات بات محاسبية ملائمة نتيجة تلافيها لعيوب التئ التكلفة التاريخية،

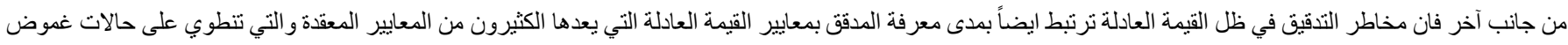

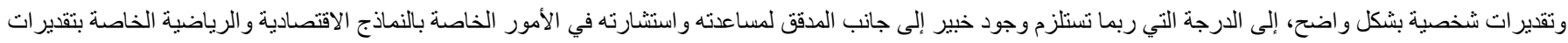

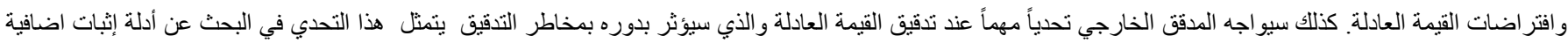
عن معقولية الافتر اضات وملائمة نماذج القياس المستخدمة في تقدير القيمة العادلة، فلن يكتفي بأدلة الإثبات التقليدية كما في التكلفة التاريخية، بل يحاول البحث عن أدلة جديدة تساعده في

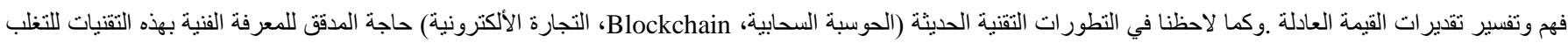
على مخاطر التدقيق المستجدة و المرتبطة بهذه التقنيات، فالكلام ذاته ينطبق على القيمة العادلة وضرورة تمتعه بمعرفة فنية خاصة بتقدير ات القيمة العادلة بالإضافة إلى مساعدة الخبير. 4.2

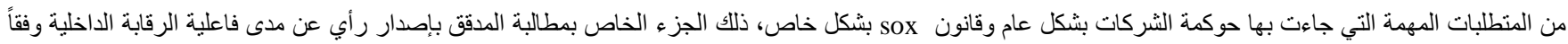

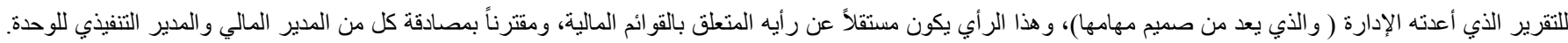

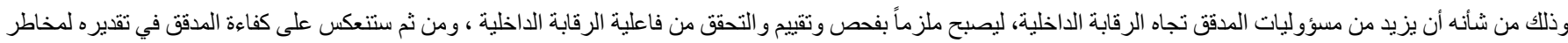

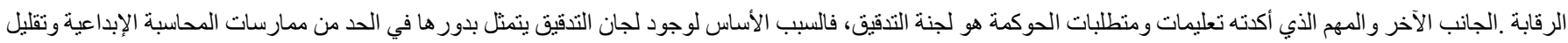
حالات الغش والتلاعب ،التي أدت سابقاً إلى زيادة حالات الإخفاق أو الإنهيار المالي للشركات. وقد جعلت الحوكمة لجنة التدقيق الجهة الوحيدة والمباشرة المسؤولة عن تعيين المدقق

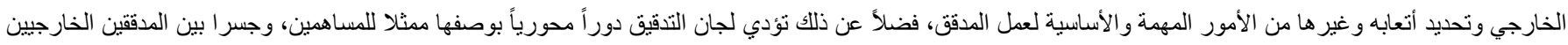

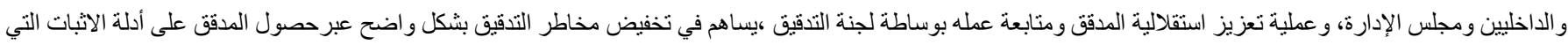

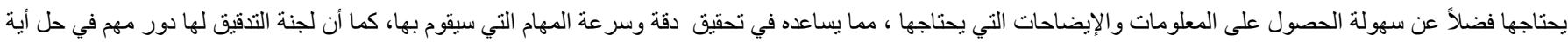

مشكلة أو سوء فهم بين المدقق و الإدارة، مما ينعكس على جودة عمل المدقق ومن التأثير بمخاطر التدقيق.

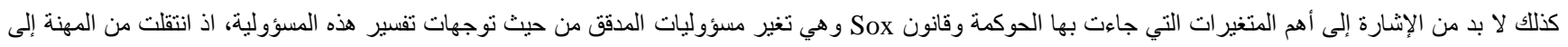

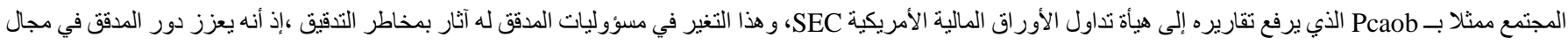
تقدير و اكتشاف مخاطر التدقيق ،وقد أصدر pcaob مجموعة معايير خاصة بمخاطر التدقيق والاستجابة لها. من جانب آخر وفي خطوة منه لمو اكبة منطلبات الحوكمة أصدر الاتحاد

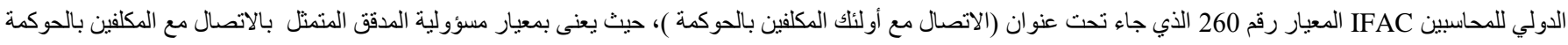

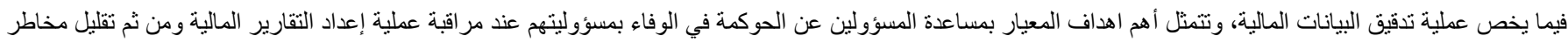
الأخطاء الجو هرية للبيانات المالية.

( 5 1. ترتبط التطور ات المهنية المؤثرة بمخاطر التدقيق بانعكاسات الأزمات المالية على مهنة المحاسبة والتدقيق و التي أهمها القيمة العادلة وحوكمة الشركات. 2. ارتبطت التطور ات التقنية المؤثرة بمخاطر التدقيق بأدوات التحول الرقمي التي اعتمدت عليها الوحدات في أعمالها، وأهمها الحوسبة السحابية وسلسلة الكتل (blockchain) و التجارة الألكترونية. 3.

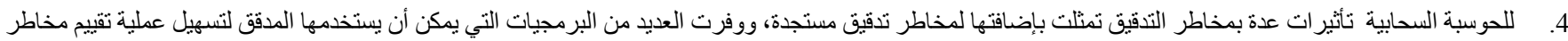
التدقيق.

5. الهدف الأساس للتدقيق في بيئة الحوسبة السحابية هو التحقق و التأكد من مدى وجود سياسة مناسبة حول الاستعانة بالحوسبة السحابية لاى الوحدة الخاضعة للتدقيق.

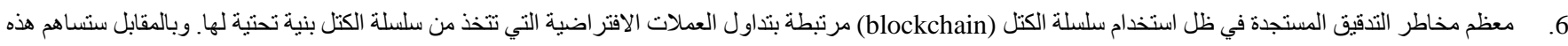
التقنية في توفير تقارير تدقيق أكثر دقة كما ستساعد في انخفاض مخاطر التدقيق، عبر القيام بالتحقق التلقائي للعديد من المعاملات بوقت قياسي.

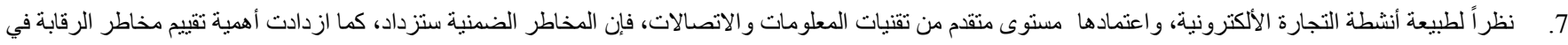

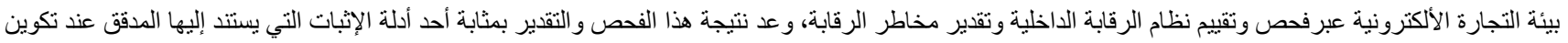
رأيه حول أنشطة التجارة الأكترونية. 
Waheed R. et.al., Journal of Techniques, Vol. 3, No. 1, March 31, 2021, Pages 1- 12

$$
\begin{aligned}
& 8 \text { 8. لقد فرضت التطور ات التقنية على المدققين ضرورة تأهيل مهني وعلمي إضافي خاص بطبيعة هذه التقنيات ومهار ات جديدة لمو اجهة مخاطر التدقيق المستجدة المرتبطة بها، فهذه } \\
& \text { التطور ات التقنية تعد تحدياً جديداً للمدققين وفي الوقت نفسه تمثل مدخلاً لتطوير الخدمات التي يقدمونها. } \\
& 9 \text { 9. إن مخاطر التدقيق في ظل القيمة العادلة ارتبطت بالتقدير ات والافتر اضات التي تعد الأساس لقياس القيمة العادلة، والتي تترك مجالاً بصورة لها اثار ها في احتمالية زيادة مخاطر } \\
& \text { التدقيق ووجود مخاطر جديدة، كما أنها ألزمت المدقق بضرورة تمتعه بمعرفة فنية، خاصة بتقدير ات القيمة العادلة بالإضافة إلى مساعدة الخبير. } \\
& \text { 10. لحوكمة الثركات دور مهم في تخفيض مخاطر التدقيق عبر التعليمات والمبادئ التي جاءت بها الحوكمة والأدوار التي أُسندت للجان التدقيق. } \\
& \text { 6 } \\
& \text { 1. العمل على تأهيل المدققين علمياً وعملياً وبما يتناسب مع التطورات التقنية وخاصة الحوسبة السحابية وسلسلة الكثل عبر التذريب، والنشرات التثقيفية، فضلاً عن تحديث مناهج } \\
& \text { التعليم المحاسبي. } \\
& \text { 2. العمل على توجيه المدقفين نحو الاستفادة من التطور ات التقنية بما يخدم المهنة ويطور هانحو الأفضل عبر إصدار المعايير الخاصة بتأثير الحوسبة السحابية وسلسلة الكتل والتجارة } \\
& \text { الألكترونية وتوظيفها في الحد من مخاطر التدقيق. } \\
& \text { 3. العمل على زيادة معرفة المدققين بمعايير القيمة العادلة والتي تعد من المعايير المعقدة التي تتطوي على حالات غموض وتقديرات شخصية، مما يساعد المدقق في البحث عن أدلة } \\
& \text { إثبات إضافية عن معقولية الافتر اضات وملائمة نماذج القياس المستخدمة في تقدير القيمة العادلة. } \\
& \text { الاستفادة من التجارب الدولية والعربية في مجال حوكمة الشركات وتوظيفها في الحد من مخاطر التدقيق. }
\end{aligned}
$$

1

[1] Ben Halpert (2011), Auditing Cloud Computing, Published by John Wiley \& Sons, New Jersey, United States of America .

[2] Emanuel Ferreira Coutinho \& Fl_avio R. \& C. Sousa_Paulo A. L. Rego _ Danielo \& G. Gomes _ Jos_e N. de Souza (2014), Elasticity in Cloud Computing: A Survey, https://www.researchgate.net/publication/268685775

[3] Hussain AlJahdali, Abdulaziz Albatli, Peter Garraghan, Paul Townend, Lydia Lau, Jie Xu (2014), Multi-Tenancy in Cloud Computing, https://www.researchgate.net/publication/260305189

[4] Imad Jaber Ramadan (2017), a proposed accounting approach to rationalize government performance expenditures for electronic services in light of cloud computing (case study), $\mathrm{PhD}$ thesis, Faculty of Commerce, Cairo University.

[5] NIST(2011), The NIST Definition of Cloud Computing, www.nvlpubs.nist.gov/nistpubs/Legacy/SP/nistspecialpublication800-1pdf

[6] INTOSAI (2014), Information Technology Audit Manual for Supreme Audit Institutions, translated by: Kuwait Audit Bureau, https://www.intosaicommunity.net >wgita> it-audit-handbook-arabic-version

[7] Alaa Abdulwahid Thanoon Taha (2018), Cloud Auditing: The Contemporary Millennium Model for Auditing Accounting Information Systems, Tikrit Journal of Administrative and Economic Sciences, Volume (4), Issue (44), Part (1), Faculty of Leasing and Economics, Tikrit University.

[8] Hoda Hussien Mohamed (2017), The Role and Responsibility of the External Auditor towards the Cloud Computing (An Empirical Study), Master Thesis, faculty of commerce and business administration, helwan university .

[9] Chris Davis \&Mike Schiller \& Kevin Wheeler(2015), IT Auditing: Using Controls to Protect Information Assets , Second Edition ,Mcgraw-hill, New York.

[10] IAASB (2019), ISA 315 (Revised 2019): Conforming and Consequential Amendments to Other International Standards Arising from ISA 315 (Revised 2019), www.iaasb.org/publications/isa-315-revised-2019-identifying-and-assessing-risks-material-misstatement

[11] unctad(2019), Digital economic report, https://unctad.org/en/pages/PublicationWebflyer.aspx?publicationid=2466

[12] Robby Houben \& Alexander Snyers(2018) , Cryptocurrencies and blockchain , European Parliament, http://www.europarl.europa.eu/cmsdata/150761/TAX3\%20Study\%20on\%20cryptocurrencies\%20and\%20blockchain.pdf

[13] Cpa \& aicpa(2017), Blockchain Technology and Its Potential Impact on the Audit and Assurance Profession, https://www.aicpa.org

[14] Michael Crosby\&Nachiappn \&Pradhan Pattanayak(2015)BlockChain Technology, Sutardja Center for Entrepreneurship \& Technology Technical Report,Berkeley University, California, http://bit.ly/2a0UB7R.

[15] Mona Hassan Al-Sharqawi (2019), an analytical study of the effect of the use of blockchain technology in the accounting environment and its reflection on various business sectors, Journal of Accounting Thought, Volume (23), Issue (1), Faculty of Commerce, Ain Shams University.

[16] ICAEW (2018), Blockchain and the Future of Accountancy, http://www.icaew.com/itfac

[17] Ihab Khalifa (2018), Blockchain, The Next Technology Revolution in the World of Finance and Management, Future Research and Studies Center, United Arab Emirates, https://futureuae.com/media/Ehabpdf_d1f747f1-7ba7-4390-bd3f-918c5dbf6ead.pdf

[18] Sanjeev K. Bansal \& Roopali Batra \& Nikita Jain(2018), Blockchain The Future Of Accounting, The Management Accountant journal, vol(53), No(6), The Institute Of Cost Accountants Of India, https://icmai.in/icmai/

[19] Ayman Muhammad Nakhal (2020), The Impact of Using Blockchain Technology on the Responsibility of the Auditor, Accounting Thought Journal, Volume (24), Issue (1), Faculty of Commerce, Ain Shams University.

[20] Zeyad Hashim Alsaqa \& ali ibrahim hussein \& saddam mohammed mahmood(2019), The Impact of Blockchain on Accounting Information Systems, Journal of Information Technology Management, vol(11), No(3), https://www.researchgate.net/publication/338396456 
[21] Abdulwahid Ghazi Mohammed (2012), The Impact of Technical and Professional Developments on External Auditing, Fifth Scientific Conference (Entrepreneurship and Business Intelligence in Iraqi Organizations), College of Business and Economics, University of Mosul, 26-27 / December, Iraq.

[22] Nazem Hassan Rashid (2011), The role of the auditor in imparting confidence in accounting data published on the Internet in the ecommerce environment, Tikrit Journal of Administrative and Economic Sciences, Volume (7), Issue (23), College of Administration and Economics, Tikrit University.

[23] Ziad Hashem Al-Sakka, Waheed Mahmoud Ramo and Qassem Mohsen Al-Hubaity, The Impact of E-commerce on Auditing Standards in the Light of the Digital Economy https://www.researchgate.net/publication/322626720_tathyr_altjart_alalktrwnyt_ly_mayyr_altdqyq_fy_zl_alaqtsad_alrqmy

[24] Ibrahim Yaqoub Ismail and Abbas Ibrahim Adam Al-Tijani (2019), Review of Fair Value Estimates and their Impact on the Appropriateness and Reliability of Accounting Information, Field Study, The Comprehensive Multidisciplinary Electronic Journal, Issue (19), https://www.eimj.org/

[25] Rahisuddin Saifi(2018),Blockchain and its impact on Auditing and Assurance profession,http://www.asa.in/filedownload?file=\%2FUpload\%2FfldInsights $\% 2$ FBlockchain

[26] richard c. kloch \& simon j. little(2018),blockchain and internal audit,https://theiia.fi/wp-content/uploads/2019/08/iaf-blockchain-internalaudit-july-2019-070119-2.pdf

[27] CPA(2018),Audit Considerations Related to Cryptocurrency Assets and Transactions, Chartered Professional Accountants of Canada, https://www.cpacanada.ca/en/business-and-accounting-resources/audit-and-assurance/canadian-auditing-standardscas/publications/cryptocurrency-audit-considerations

[28] CPAB \& CCRC(2018) , Auditing in the Crypto-Asset Sector , http://www.cpabccrc.ca/Documents/News\%20and\%20Publications/Auditing\%20in\%20the\%20Crypto-Asset\%20Sector.pdf

[29] Yunji Liang \& Lijun Bu(2016), Study on the audit risk under the environment of e-commerce, 3rd International Conference on Education Management and Computing Technology, Hangzhou, China, www.researchgate.net/publication/303481598_Study_on_the_audit_risk_under_the_environment_of_e-commerce

[30] Nasreen Muhammad Saeed Al-Malih (2009), The Impact of Electronic Commerce on Review Risk, An Empirical Study, Master Thesis, Faculty of Commerce, Menoufia University.

[31] Muhammad Bahaa Al-Din Ibrahim Ahmed (2010), Towards an Integrated Approach proposed for External Review of Electronic Commerce Activities to Reduce Review Risk: An Applied Theory Study, Journal of Accounting Thought, Volume (14), Issue (2), Faculty of Commerce, Ain Shams University.

[32] Mahmoud Ibrahim, Hasna Attia, Abdullah Wasow (2015), The Impact of the Audit Risk Structure on the Evaluation of Internal Control in Light of the Application of the Fair Value Measurement Standard, The Egyptian Journal of Commercial Studies, Volume (39), Issue (3), Faculty of Commerce, Mansoura University.

[33] Ali Muhammad Ali Al-Sayyad (2016), a proposed framework for reviewing fair value accounting estimates using risk-based review, Journal of Accounting and Auditing, Volume (4), Faculty of Commerce, Beni Suef University. 